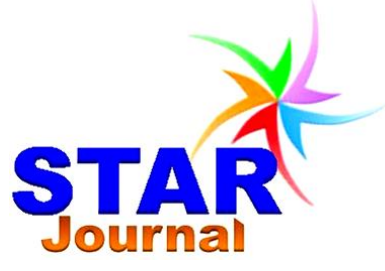

ISSN: 2226-7522(Print) and 2305-3327 (Online) Science, Technology and Arts Research Journal

July-Sep 2013, 2(3): 93-104

www.starjournal.org

Copyright@2013 STAR Journal. All Rights Reserved

Original Research

\title{
Ecological Phytogeography: A Case Study of Commiphora Species
}

\author{
Teshome Soromessa
}

Center for Environmental Science, Addis Ababa University, Post Box No: 1176, Addis Ababa Ethiopia

\begin{tabular}{|c|c|}
\hline Abstract & Article Information \\
\hline \multirow{4}{*}{$\begin{array}{l}\text { The present paper stipulated phytogeography, ecological ranges, possible origin and } \\
\text { migratory route of Commiphora Jacq. species. Data were gathered from the field, } \\
\text { herbarium and secondary sources. Information on distribution, altitude and soil } \\
\text { preferences were compiled and aggregated together. Phytogeographical aspect of } \\
\text { the group has been analyzed using Brooks's parsimony analysis (1990) which was } \\
\text { done by tabulating flora regions versus the species under consideration where the } \\
\text { matrix has been filled as either presence or absence. The result of data on } \\
\text { phytogeography showed three patterns of distribution. Based on the plate tectonic } \\
\text { theory, evolution and diversification of most angiosperm families into consideration, } \\
\text { the origin of Commiphora has been discussed in details. It was recommended that } \\
\text { the migratory route of Commiphora still requires further investigation and needs to } \\
\text { be corroborated with data on the age of the genus and that of the concept of plate } \\
\text { tectonic theory. }\end{array}$} & \begin{tabular}{ll}
\multicolumn{2}{l}{ Article History: } \\
Received $: 28-07-2013$ \\
Revised $: 21-09-2013$ \\
Accepted $: 24-09-2013$
\end{tabular} \\
\hline & $\begin{array}{l}\text { Keywords: } \\
\text { Commiphora } \\
\text { Ecological Ranges } \\
\text { Phytogeography }\end{array}$ \\
\hline & $\begin{array}{l}{ }^{*} \text { Corresponding Author: } \\
\text { Teshome Soromessa }\end{array}$ \\
\hline & $\begin{array}{l}\text { E-mail: } \\
\text { teshome.soromessa@aau.edu.et }\end{array}$ \\
\hline
\end{tabular}

\section{INTRODUCTION}

The genus Commiphora Jacq. is one of the most diverse genera of the family Burseraceae. It is largely represented in Africa where it is confined to arid and semi-arid areas. Commiphora also occurs in Iran, Pakistan, Peninsula India, Sri Lanka and Brazil. The genus is co-dominant with Acacia over the huge areas of the Horn of Africa and supports the large livestock populations of pastoral and agro pastoral communities. Of the 190 or so species in the genus, more than half of the species are native to the Horn of Africa. This might support the notion that the center of genetic diversity of the genus is in this part of the world.

In earlier days attempts have been made to circumscribe the genus, among others, Berg (1862), Engler (1898, 1904, 1910, 1912, 1931 and 1931), Sprague (1927), Chiovenda (1932), Burtt (1935), Leenhouts (1956), Wild (1959 and 1963), Van der Walt (1973 and 1986), Gillett (1980 and 1991) and Boulos (2000) are the ones to be mentioned. However, information on the phytogeography, niche ranges, possible origin and migratory route of the group has not been treated in the past. Thus, the present paper aimed at dealing with the aforementioned information taking the genus Commiphora in to consideration.

\section{MATERIAL AND METHODS}

Studies on NE and E Tropical African species of Commiphora were made (Table 1 for the nomenclature of taxa). Data were gathered from the intensive field works, herbarium specimens with the consultations of various literatures. Information on distribution, altitude and soil preferences were compiled and aggregated together. Phytogeographcal aspect of the group has been analyzed using Brooks's parsimony analysis (1990). This was done by tabulating flora regions versus the species under consideration where the matrix has been filled as either presence or absence.

The geographical areas included under the present investigation were NE (North East which refers to Ethiopia and Eritrea) and $E$ (East referring to Kenya, Tanzania and Uganda) Tropical Africa. Because of the transgressing nature of Commiphora species to the adjacent Somalia, Somalia was also included in the map for discussion purposes. The description of the regional floristic divisions of the 
Teshome Soromessa

flora areas (i.e., Ethiopia, Eritrea, East Africa and Somalia) followed Hedberg and Edwards (1989), Polhill (1988) and Thulin (1999) as given in Figure 1.

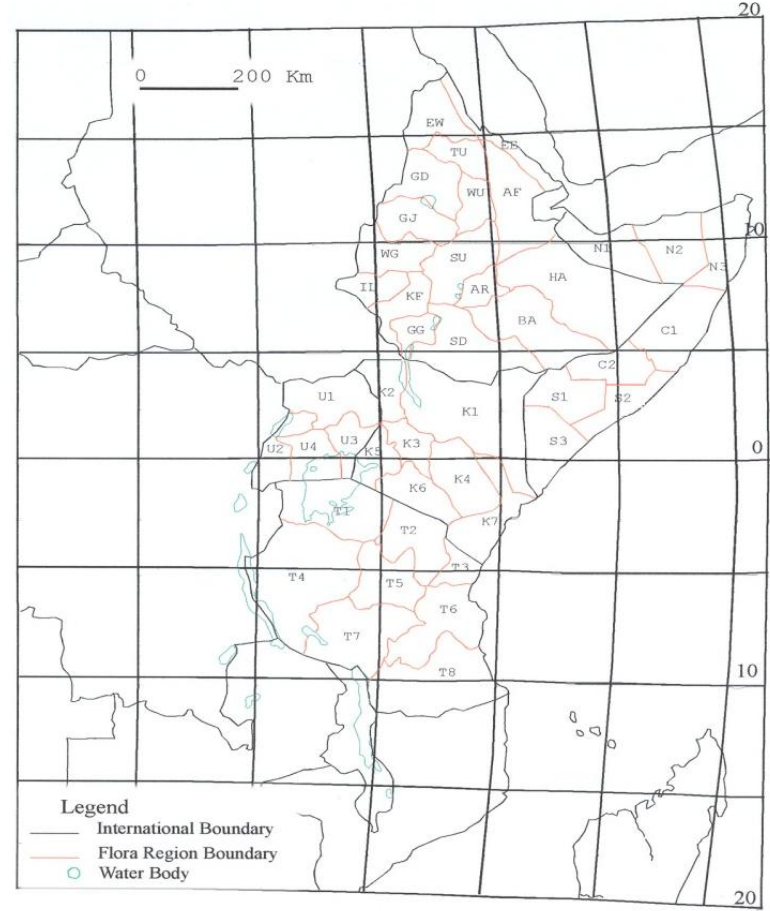

Figure 1: Regional floristic divisions of Ethiopia, Eritrea, East Africa and Somalia.

\section{RESULTS AND DISCUSSION}

\section{Phytogeograpy}

The majority of the species of Commiphora are essentially pan tropical in their distribution extending from Brazil via Tropical Africa to India and Pakistan with their high concentration in the Horn of Africa. On the account of the subdivision of African phytochoria of White (1983), the genus is found distributed in the majority of the regional centers of endemism, regional mosaic and transitional zones, with the highest genetic diversity in the SomaliaMasai regional center of endemism. Accordingly Commiphora species are found in Zambezian regional center of endemism, the Sudanian regional center of endemism, the Somalia-Masai regional center of endemism, the Karoo-Namib regional center of endemism, the Zanzibar-Inhambane regional mosaic, the Kalahari-highveld regional transitional zone, the Tongaland-Pondoland regional mosaic, the Sahel regional transition zone and the Sahara regional transition zone. However, some species like $C$. myrrha is transcontinental and extends its distribution to Arabia and India.

Out of the species of NE and E Tropical African Commiphora currently dealt with, only a few of them are out of the Somalia-Masai regional center of
Sci. Technol. Arts Res. J., July-Sep 2013, 2(3): 93-104

endemism. For example, C. africana has a wider distribution through out the continent. It occurs in Zambezian region, Sudanian region, Somalia-Masai region, Kalahari-Highveld region, Sahel region and the Sahara region. C. africana has been reported from more that 30 countries in Africa (van der Walt, 1975). Others like C. schimperi, C. gileadensis, $C$. quadricincta, C. habessinica, C. kataf and C. myrrha transgress beyond the continent and occur in Arabia having disjunct distribution. Two species, namely $C$. monoica and $C$. suffruticosa are endemic to Ethiopia; another two species, C. oblongifolia and $C$. ovalifolia are endemic to Kenya; six species, $C$. madagascarensis, C. hornbyi, C. acuminata, C. engleri, $C$. spathulata and $C$. stolonifera are endemic to Tanzanian flora (see Fig. 2).

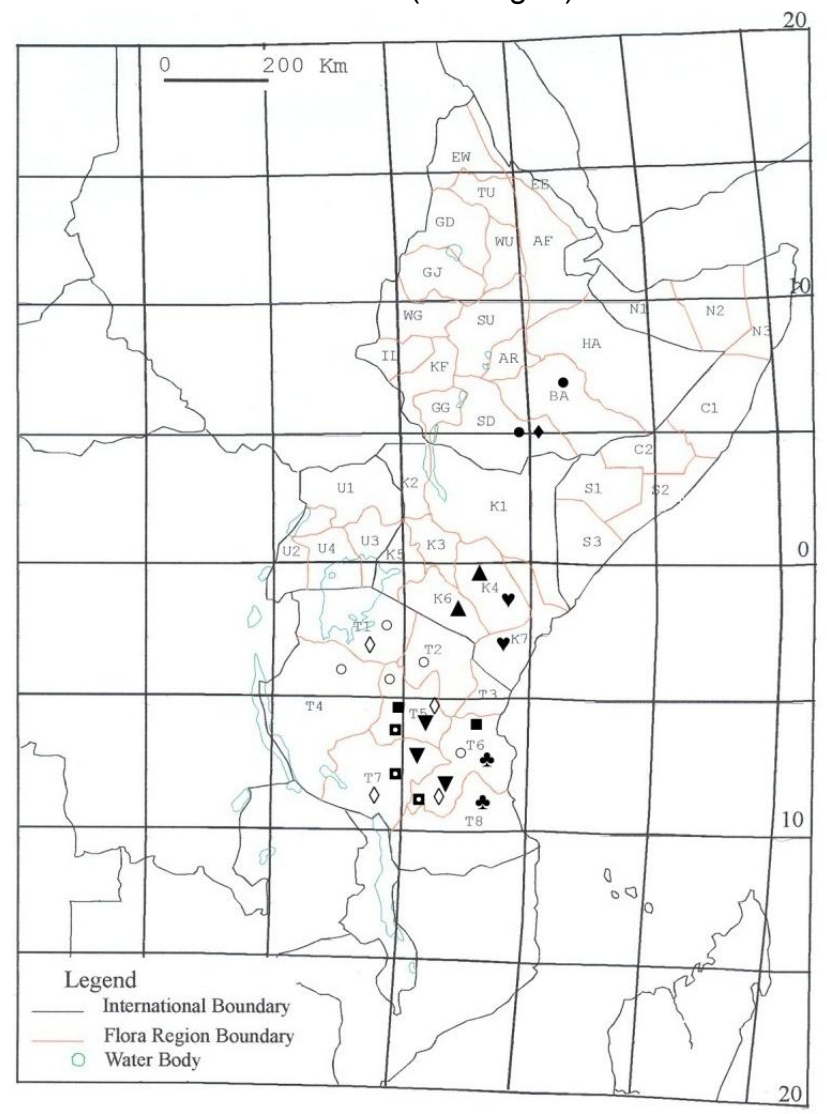

Figure 2: Distribution of endemic species of Commiphora in NE and East Tropical Africa in different flora regions. The symbols indicated in the map represent different species: - - C. monoica, - $C$. suffruticosa, $\bullet$ - C. oblongofolia, $\boldsymbol{\Delta}-\mathrm{C}$. ovalifolia, - C. madagascarensis, $\nabla$ - C. hornbyi, - $-C$. acuminata, $\circ-C$. engleri, 口 - C. spathulata,. $\diamond$ - C. stolonifera.

One approach of tracing the phytogeographic aspect of a given group is using Brooks's parsimony analysis (1990). In this analysis flora regions (areas) 
Teshome Soromessa

versus species were tabulated on the data matrix and this has been generated from Table 1. Within the developed matrix absence and presence were considered. The data matrix was analyzed using PAUP Swofford (2002). The result obtained is reproduced in Figure 3.

The present gross distributional data for Commiphora of NE and E Tropical Africa (Figure 3)
Sci. Technol. Arts Res. J., July-Sep 2013, 2(3): 93-104

shows three types of pattern. The first type is the EW-GG group that is characterized by wide spread distributional pattern and know to have few species of Commiphora. In this group few Commiphora species are distributed such as $C$. africana, $C$. habessinica, $C$. edulis and $C$. schimperi that normally occurs elsewhere in the different areas.

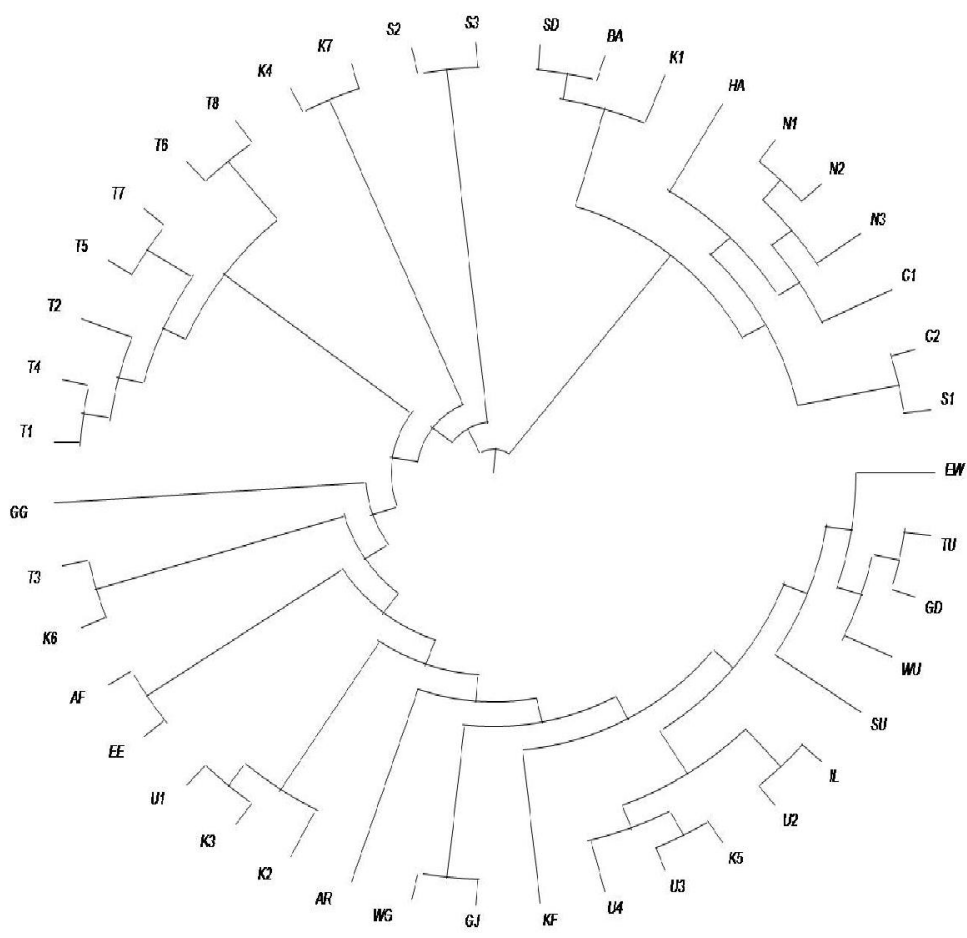

Figure 3: The areagram obtained using BPA.

The second group, T1-S3 (Figure. 3), is partly Tanzanian and characterized by group with very limited distributional pattern. Some species occurring in these areas are more or less endemic (such as C. madagascarensis), others are transgressing ones that transgress down to Southern African region and still some display very limited pattern of distribution. The transgressing species include C. mollis, C. mossambicensis and C. mombassensis.

The third group, SD-S1 group (Figure. 3), is the pattern displayed by most Commiphora species. About $70 \%$ of Commiphora occurring in NE and E Tropical Africa are found distributed in these regions. Examples to this pattern are $C$. hildebrabdtii and $C$. ogadensis. This perhaps seems to be the appropriate environment that suits many Commiphora species to survive, diversify and invade the region. Except for the political boundaries these areas are almost adjacent to one another and sharing similar ecological niche that support similar life forms.

\section{Possible Origin and Migratory Route}

The phylogenetic study of Commiphora species reported by Teshome (2005) presumably indicated that the primitive group with in the sections belongs to section Cupulares which contains $C$. eralngeriana, C. monoica, C. erosa, C. eminii, $C$. hornbyi, C. unilobata and C. zanziberica. In addition to this evidence, the Horn of Africa harbors all sex forms that occur in the genus; i.e. hermaphrodite, monoecious and dioecious with the possible intermediate groups and the xerophytic and mesophytic species of Commiphora also occur in the NE and E Tropical Africa. Apparently it is possible to make the presumptuous assumption that the Horn of Africa is the origin of the group, with more emphasis to the NE of Africa. Although Wild (1965), didn't explicitly pointed out about the origin of the genus, he indicated that the occurrence of very dry interpluvial periods in Africa put pressure on the genus Commiphora to produce xerophytic species now well represented in NE and SW Africa, some of which migrated without difficulty through 
Teshome Soromessa

Sci. Technol. Arts Res. J., July-Sep 2013, 2(3): 93-104

Table 1: Altitudinal ranges, average annual rainfall, pedological preference and distribution of Commiphora species.

\begin{tabular}{|c|c|c|c|c|c|c|c|c|c|}
\hline Scientific name & $\begin{array}{l}\text { Altitudinal } \\
\text { range in } \mathrm{m}\end{array}$ & $\begin{array}{c}\text { Annual } \\
\text { Rainfall in } \\
\mathbf{m m}\end{array}$ & $\begin{array}{l}\text { Pedological } \\
\text { preference }\end{array}$ & NE & K1-7 & T1-8 & U1-4 & $\mathbf{S}$ & CWS-Africa \\
\hline C. acuminata Mattick & $750-900$ & $600-750$ & rocky slopes & & & 56 & & & \\
\hline C. africana Engl. & $2-2100$ & $200-850$ & $\begin{array}{l}\text { sandy to } \\
\text { loamy, } \\
\text { recent lava } \\
\text { flows }\end{array}$ & $\begin{array}{l}\text { EE, AF, EW, TU, } \\
G D, W U, S U, \\
K F, G G, S D, B A \text {, } \\
H A\end{array}$ & K1-7 & $\mathrm{T} 1-8$ & 123 & N12C1S3 & $\begin{array}{l}\text { Tropical and } \\
\text { southern } \\
\text { Africa }\end{array}$ \\
\hline $\begin{array}{l}\text { C. alaticaulis Gillett } \\
\text { and Vollesen }\end{array}$ & $300-1200$ & $200-450$ & $\begin{array}{l}\text { sandy, rocky } \\
\text { lime stone }\end{array}$ & $\mathrm{SD}, \mathrm{BA}, \mathrm{HA}$ & 2 & & & $\mathrm{~N} 2 \mathrm{C} 2 \mathrm{~S} 1 \mathrm{~S} 3$ & \\
\hline C. albiflora Engl. & $700-1000$ & $200-350$ & $\begin{array}{l}\text { sandy, rocky } \\
\text { lime stone }\end{array}$ & SD,BA & & & & $\mathrm{C} 2 \mathrm{~S} 2$ & \\
\hline C. baluensis Engl. & $600-1700$ & $450-800$ & $\begin{array}{l}\text { sandy, black } \\
\text { cotton, rocky } \\
\text { slopes }\end{array}$ & $\begin{array}{l}E F, A F, S U, A R, \\
G G, S D, B A, H A\end{array}$ & 1467 & 23 & & & Sudan \\
\hline $\begin{array}{l}\text { C. boranensis } \\
\text { Vollesen }\end{array}$ & $750-1450$ & $250-450$ & $\begin{array}{l}\text { rocky } \\
\text { limestone, } \\
\text { black cotton }\end{array}$ & $\mathrm{SD}, \mathrm{BA}, \mathrm{HA}$ & 1 & & & N1S1S2 & \\
\hline C. bruceae Chiov. & $150-1100$ & $200-280$ & alluvial sand & $\mathrm{SD}, \mathrm{BA}, \mathrm{HA}$ & 127 & 3 & & $\begin{array}{l}\mathrm{N} 1-3 \mathrm{C} 1-2 \\
\mathrm{~S} 1-3\end{array}$ & \\
\hline C. caerulea B.D. Burtt & $500-1500$ & $450-700$ & sandy & & & 14567 & & & $\begin{array}{l}\text { Malawi, } \\
\text { Zimbabwe, } \\
\text { Botswana }\end{array}$ \\
\hline C. campestris Engl. & $50-1200$ & $230-800$ & $\begin{array}{l}\text { sandy, rocky } \\
\text { lava hills, } \\
\text { alluvial } \\
\end{array}$ & SD & 1467 & 2356 & & C2S1-3 & \\
\hline C. chaetocarpa Gillett & 200 & 230 & sandy & & 1 & & & $\mathrm{C} 2$ & \\
\hline C. ciliata Vollesen & $700-1250$ & $300-450$ & $\begin{array}{l}\text { rock lime } \\
\text { stone hills }\end{array}$ & $\mathrm{SD}, \mathrm{BA}$ & 1 & & & $\mathrm{C} 1-2 \mathrm{~S} 1$ & \\
\hline C. confusa Vollesen & $150-1300$ & $300-700$ & $\begin{array}{l}\text { sandy, rocky } \\
\text { slopes }\end{array}$ & GG, SD, BA & 12467 & 3 & & & \\
\hline $\begin{array}{l}\text { C. corrugata Gillett } \\
\text { and Vollesen }\end{array}$ & $300-1450$ & $250-380$ & $\begin{array}{l}\text { rocky } \\
\text { limestone }\end{array}$ & $\mathrm{SD}, \mathrm{BA}, \mathrm{HA}$ & 1 & & & $\mathrm{C} 2 \mathrm{~S} 1$ & \\
\hline C. cyclophylla Chiov. & $300-800$ & $200-300$ & $\begin{array}{l}\text { sandy, } \\
\text { limestone } \\
\text { slope } \\
\end{array}$ & $\mathrm{SD}, \mathrm{BA}, \mathrm{HA}$ & 1 & & & N2-3C1-2 S1 & \\
\hline C. danduensis Gillett & $450-1600$ & $250-700$ & sandy & SD & 1 & & & & \\
\hline C. edulis Engl. & $2-1400$ & $350-900$ & $\begin{array}{l}\text { sandy, rock } \\
\text { slopes }\end{array}$ & $\mathrm{SD}, \mathrm{GG}$ & 123467 & 1234567 & 1 & S1-3 & $\begin{array}{l}\text { Zambia, } \\
\text { Malawi, } \\
\text { Mozambique, } \\
\text { Botswana, } \\
\text { South Africa }\end{array}$ \\
\hline C. ellenbeckii Engl. & $300-1200$ & $230-350$ & $\begin{array}{l}\text { sandy, rocky } \\
\text { slopes }\end{array}$ & $\mathrm{SD}, \mathrm{BA}, \mathrm{HA}$ & 1 & & & $\begin{array}{l}\mathrm{N} 1-3 \mathrm{C} 1-2 \\
\mathrm{~S} 1-2\end{array}$ & \\
\hline C. eminii Engl. & $100-1750$ & $550-1200$ & $\begin{array}{l}\text { rocky hills, } \\
\text { limestone }\end{array}$ & & 467 & 12345678 & & & $\begin{array}{l}\text { Zambia, } \\
\text { Malawi, } \\
\text { Mozambique }\end{array}$ \\
\hline C. engleri Guillaumin & $870-1650$ & $450-800$ & rocky hills & & & 12456 & & & \\
\hline C. erlangeriana Engl. & $250-700$ & $230-250$ & $\begin{array}{l}\text { rocky } \\
\text { limestone, } \\
\text { gypsum } \\
\text { slope } \\
\end{array}$ & $\mathrm{SD}, \mathrm{BA}$ & 1 & & & $N 1-3 C 1-2 S 1$ & \\
\hline C. erosa Vollesen & $200-400$ & $230-300$ & $\begin{array}{l}\text { gypsum hills, } \\
\text { alluvium } \\
\text { limestone }\end{array}$ & $\mathrm{SD}, \mathrm{BA}$ & 17 & & & $\mathrm{C} 2 \mathrm{~S} 1-3$ & \\
\hline $\begin{array}{l}\text { C. fulvotomentosa } \\
\text { Engl. }\end{array}$ & $200-1050$ & $800-1000$ & $\begin{array}{l}\text { rocky } \\
\text { outcrops }\end{array}$ & & & 68 & & & \\
\hline C. gileadensis C. Chr. & $150-750$ & $200-350$ & $\begin{array}{l}\text { rocky hills, } \\
\text { sand dunes }\end{array}$ & $\mathrm{EE}, \mathrm{HA}$ & & & & N1-3C1-2 S1 & Egypt, Sudan \\
\hline C. glandulosa Schinz & $5-950$ & $650-950$ & rocky slopes & & & 568 & & & $\begin{array}{l}\text { Zaire, Angola, } \\
\text { Zambia, }\end{array}$ \\
\hline
\end{tabular}




\begin{tabular}{|c|c|c|c|c|c|c|c|c|c|}
\hline & & & & & & & & & $\begin{array}{l}\text { Malawi, } \\
\text { Mozambique, } \\
\text { Namibia, } \\
\text { Botswana, } \\
\text { Zimbabwe, } \\
\text { South Africa }\end{array}$ \\
\hline C. gowlello Sprague & $350-1400$ & $200-350$ & $\begin{array}{l}\text { sandy, stony } \\
\text { soil }\end{array}$ & $\mathrm{SD}, \mathrm{BA}, \mathrm{HA}$ & & & & $\mathrm{N} 1-2 \mathrm{C} 1-2 \mathrm{~S} 1$ & \\
\hline C. gracilispina Gillett & $350-400$ & 230 & sandy loam & & 1 & & & $\mathrm{~N} 1-3 \mathrm{C} 2 \mathrm{~S} 2$ & \\
\hline C. guidottii Chiov. & $250-400$ & $230-250$ & $\begin{array}{l}\text { gypsum soil, } \\
\text { stony slope }\end{array}$ & SD, BA & & & & $\mathrm{N} 2-3 \mathrm{C} 1-2 \mathrm{~S} 1$ & \\
\hline C. gurreh Engl. & $750-1500$ & $200-500$ & $\begin{array}{l}\text { black cotton, } \\
\text { rocky lime } \\
\text { stone }\end{array}$ & $\mathrm{SD}, \mathrm{BA}$ & 1 & & & $\begin{array}{l}\mathrm{N} 1-3 \mathrm{C} 1-2 \\
\mathrm{~S} 1-2\end{array}$ & \\
\hline C. habessinica Engl. & $500-1900$ & $300-900$ & $\begin{array}{l}\text { sandy to } \\
\text { loamy, black } \\
\text { cotton }\end{array}$ & $\begin{array}{l}\text { EE, EW, TU, } \\
\text { WU, GD, GJ, SU, } \\
\text { WG, IL, GG, SD, } \\
\text { BA, HA }\end{array}$ & 123467 & 123567 & 12 & N1N2 & $\begin{array}{l}\text { Zambia, } \\
\text { Malawi, Zaire, } \\
\text { Rwanda, } \\
\text { Burundi, } \\
\text { Djibouti }\end{array}$ \\
\hline C. hildebrandtii Engl. & $850-1500$ & $300-550$ & $\begin{array}{l}\text { rocky lime } \\
\text { stone slopes }\end{array}$ & $\mathrm{SD}, \mathrm{BA}$ & & & & $\mathrm{N} 1-3 \mathrm{C} 2 \mathrm{~S} 1-2$ & \\
\hline C. hodai Sprague & $400-650$ & $230-250$ & $\begin{array}{l}\text { red sandy } \\
\text { soil }\end{array}$ & $\mathrm{HA}$ & & & & $\mathrm{N} 1-2 \mathrm{C} 1-2 \mathrm{~S} 1$ & \\
\hline C. hornbyi B.D. Burtt & $380-1220$ & $450-750$ & sandy soil & & & 567 & & & \\
\hline C. horrida Chiov. & 500 & 250 & sandy soil & $\mathrm{HA}$ & & & & $\mathrm{C} 1 \mathrm{C} 2 \mathrm{~S} 2$ & \\
\hline $\begin{array}{l}\text { C. kataf (Forssk.) } \\
\text { Engl. }\end{array}$ & $260-1650$ & $300-500$ & $\begin{array}{l}\text { drained } \\
\text { sandy soil }\end{array}$ & & 123 & 2 & & $\begin{array}{l}\text { All except } \\
\text { S3 }\end{array}$ & \\
\hline C. kua Vollesen & $90-1000$ & $230-350$ & $\begin{array}{l}\text { sandy soil, } \\
\text { rocky slopes }\end{array}$ & $\begin{array}{l}E E, A F, S D, B A, \\
H A\end{array}$ & 147 & & & $\begin{array}{l}\text { N1-3C1-2 } \\
\text { S1S3 }\end{array}$ & \\
\hline C. lindensis Engl. & $5-960$ & $600-950$ & sandy soil & & 47 & 368 & & $\mathrm{~S} 2 \mathrm{~S} 3$ & \\
\hline $\begin{array}{l}\text { C. madagascarensis } \\
\text { Jacq. }\end{array}$ & $5-660$ & $750-950$ & rocky slopes & & & 68 & & & \\
\hline C. merkeri Engl. & $700-1600$ & $300-400$ & $\begin{array}{l}\text { sandy, black } \\
\text { cotton soil }\end{array}$ & & 6 & 2 & & & \\
\hline C. mildbraedii Engl. & $450-1650$ & $500-700$ & $\begin{array}{l}\text { rocky places, } \\
\text { black cotton }\end{array}$ & BA & 147 & & & & \\
\hline C. mollis Engl. & $540-1500$ & $400-900$ & $\begin{array}{l}\text { sandy soil, } \\
\text { black cotton }\end{array}$ & & & 1245678 & & & $\begin{array}{l}\text { Zaire, Angola, } \\
\text { Zambia, } \\
\text { Malawi, } \\
\text { Mozambique, } \\
\text { Namibia, } \\
\text { Botswana, } \\
\text { Zimbabwe, S. } \\
\text { Africa }\end{array}$ \\
\hline $\begin{array}{l}\text { C. mombassensis } \\
\text { Engl. }\end{array}$ & 125 & 900 & $\begin{array}{l}\text { coastal } \\
\text { dunes }\end{array}$ & & & 8 & & & Mozambique \\
\hline C. monoica Vollesen & $1000-1400$ & $250-450$ & $\begin{array}{l}\text { rocky } \\
\text { limestone, } \\
\text { black cotton }\end{array}$ & $\mathrm{SD}, \mathrm{BA}$ & & & & & \\
\hline $\begin{array}{l}\text { C. mossambicensis } \\
\text { Engl. }\end{array}$ & $390-1660$ & $500-800$ & $\begin{array}{l}\text { sandy soil, } \\
\text { black cotton }\end{array}$ & & & 1245678 & & & $\begin{array}{l}\text { Zambia, } \\
\text { Malawi, } \\
\text { Mozambique, } \\
\text { Botswana, } \\
\text { Zimbabwe }\end{array}$ \\
\hline C. myrrha Engl. & $250-1300$ & $230-350$ & $\begin{array}{l}\text { sandy to } \\
\text { loamy, rocky } \\
\text { lava hills }\end{array}$ & $A F, S D, B A, H A$ & 1 & & & $\begin{array}{l}\mathrm{N} 1-3 \mathrm{C} 1-2 \\
\mathrm{~S} 1\end{array}$ & \\
\hline C. oblongifolia Gillett & $600-1050$ & $500-600$ & rocky hills & & 47 & & & & \\
\hline C. obovata Chiov. & $150-1050$ & $300-400$ & $\begin{array}{l}\text { rocky lime } \\
\text { stone hills }\end{array}$ & $\mathrm{SD}, \mathrm{HA}$ & 7 & & & $\mathrm{~N} 1 \mathrm{C} 2$ & \\
\hline C. oddurensis Chiov. & $400-600$ & $200-250$ & $\begin{array}{l}\text { limestone } \\
\text { slopes }\end{array}$ & & 1 & & & C1-2S1-2 & \\
\hline
\end{tabular}


Teshome Soromessa

Sci. Technol. Arts Res. J., July-Sep 2013, 2(3): 93-104

\begin{tabular}{|c|c|c|c|c|c|c|c|c|c|}
\hline C. ogadensis Chiov. & $500-1450$ & $350-650$ & $\begin{array}{l}\text { sandy soil, } \\
\text { black cotton, } \\
\text { alluvial }\end{array}$ & $\mathrm{SD}, \mathrm{BA}, \mathrm{HA}$ & 1 & & & N1-3 C2S1-2 & \\
\hline C. ovalifolia Gillett & $800-1400$ & $600-800$ & rocky slopes & & 46 & & & & \\
\hline C. paolii Chiov. & $50-700$ & $200-350$ & alluvial sand & $\mathrm{SD}, \mathrm{BA}, \mathrm{HA}$ & 147 & & & C1-2S1S3 & \\
\hline C. pedunculata Engl. & $200-1000$ & $450-800$ & sandy soil & GD, GJ, WG & & 78 & & & $\begin{array}{l}\text { Mali to } \\
\text { Sudan, } \\
\text { Malawi, } \\
\text { Zambia }\end{array}$ \\
\hline C. pteleifolia Engl. & $1-1100$ & $700-950$ & $\begin{array}{l}\text { rocky } \\
\text { escarpment }\end{array}$ & & 7 & 3468 & & & $\begin{array}{l}\text { Zaire, Zambia, } \\
\text { Mozambique }\end{array}$ \\
\hline $\begin{array}{l}\text { C. quadricincta } \\
\text { Schweinf. }\end{array}$ & $40-750$ & $200-350$ & $\begin{array}{l}\text { sandy, rocky } \\
\text { slopes, lava } \\
\text { hills }\end{array}$ & $E E, A F, E W$ & & & & & $\begin{array}{l}\text { Nigeria, Chad, } \\
\text { Sudan }\end{array}$ \\
\hline C. rostrata Engl. & $80-1350$ & $200-450$ & $\begin{array}{l}\text { sandy soil, } \\
\text { rocky slopes }\end{array}$ & $\mathrm{GG}, \mathrm{SD}, \mathrm{BA}, \mathrm{HA}$ & 147 & & & $\begin{array}{l}\mathrm{N} 1-3 \mathrm{C} 1- \\
2 \mathrm{~S} 1-2\end{array}$ & \\
\hline $\begin{array}{l}\text { C. samharensis } \\
\text { Schweinf. }\end{array}$ & $150-400$ & $150-250$ & $\begin{array}{l}\text { sandy and } \\
\text { rocky hills }\end{array}$ & $\mathrm{EE}, \mathrm{GG}$ & & & & In all & Sudan \\
\hline $\begin{array}{l}\text { C. sarandensis B.D. } \\
\text { Burtt }\end{array}$ & $750-1300$ & $350-450$ & $\begin{array}{l}\text { stony soils, } \\
\text { hard-pan } \\
\text { soils }\end{array}$ & & 1 & 1245 & & & \\
\hline C. schimperi Engl. & $430-2100$ & $450-850$ & $\begin{array}{l}\text { sandy, rocky, } \\
\text { black cotton } \\
\text { soils }\end{array}$ & $\begin{array}{l}\text { EW, TU, GD, } \\
\text { WU, SU, GJ, SU, } \\
\text { AR, GG, SD, BA, } \\
\text { HA }\end{array}$ & 123467 & 123567 & 1 & $\mathrm{~N} 1 \mathrm{C} 2$ & $\begin{array}{l}\text { Zaire, } \\
\text { Botswana, } \\
\text { Zimbabwe, } \\
\text { Mozambique, } \\
\text { S. Africa }\end{array}$ \\
\hline C. sennii Chiov. & $200-900$ & $200-400$ & alluvium soil & & 147 & & & S1S2S3 & \\
\hline C. serrata Engl. & $2-700$ & $800-950$ & $\begin{array}{l}\text { rocky } \\
\text { outcrops }\end{array}$ & & & 68 & & & Mozambique \\
\hline C. serrulata Engl. & $1000-1850$ & $350-750$ & $\begin{array}{l}\text { sandy soil, } \\
\text { black cotton, } \\
\text { basaltic soil }\end{array}$ & $\mathrm{SD}, \mathrm{BA}, \mathrm{HA}$ & & & & N1N2 & \\
\hline C. spathulata Mattick & $550-1100$ & $450-600$ & $\begin{array}{l}\text { rocky slopes, } \\
\text { red soil }\end{array}$ & & & 567 & & & \\
\hline $\begin{array}{l}\text { C. sphaerocarpa } \\
\text { Chiov. }\end{array}$ & $250-400$ & $200-250$ & $\begin{array}{l}\text { gypsum } \\
\text { slope, rocky } \\
\text { limestone }\end{array}$ & $\mathrm{SD}, \mathrm{BA}, \mathrm{HA}$ & & & & $\begin{array}{l}\mathrm{N} 1-3 \quad \mathrm{C} 1- \\
2 \mathrm{~S} 1\end{array}$ & \\
\hline $\begin{array}{l}\text { C. sphaerophylla } \\
\text { Chiov. }\end{array}$ & $400-800$ & $230-350$ & $\begin{array}{l}\text { sandy soil, } \\
\text { rocky slopes }\end{array}$ & $\mathrm{HA}$ & & & & $\begin{array}{l}\text { N1-2 C1- } \\
\text { 2S1 }\end{array}$ & \\
\hline $\begin{array}{l}\text { C. staphyleifolia } \\
\text { Chiov. }\end{array}$ & $250-400$ & $200-250$ & alluvial sand & $\mathrm{SD}, \mathrm{HA}$ & & & & $\mathrm{C} 2 \mathrm{~S} 2$ & \\
\hline $\begin{array}{l}\text { C. stolonifera B.D. } \\
\text { Burtt }\end{array}$ & $690-1260$ & $450-550$ & sandy soil & & & 1567 & & & \\
\hline $\begin{array}{l}\text { C. suffruticosa } \\
\text { Teshome }\end{array}$ & $1000-1450$ & $230-400$ & $\begin{array}{l}\text { sandy soil, } \\
\text { rocky slopes }\end{array}$ & SD & & & & & \\
\hline $\begin{array}{l}\text { C. swynnertonii B.D. } \\
\text { Burtt }\end{array}$ & $750-1100$ & $250-550$ & sandy soil & & 1 & 5 & & & \\
\hline $\begin{array}{l}\text { C. terebinthina } \\
\text { Vollesen }\end{array}$ & $200-1800$ & $300-800$ & $\begin{array}{l}\text { sandy, rocky, } \\
\text { black cotton } \\
\text { soils }\end{array}$ & $\begin{array}{l}\mathrm{KF}, \mathrm{GG}, \mathrm{SD}, \mathrm{BA}, \\
\mathrm{HA}\end{array}$ & 123467 & 2 & 1 & $\begin{array}{l}\mathrm{N} 1-3 \mathrm{C} 1- \\
2 ` \mathrm{~S} 1-3\end{array}$ & \\
\hline C. truncata Engl. & $600-1050$ & $250-400$ & $\begin{array}{l}\text { sandy, stony } \\
\text { soil }\end{array}$ & $\mathrm{HA}$ & & & & $\mathrm{N} 1-2 \mathrm{C} 1-2 \mathrm{~S} 1$ & \\
\hline C. ugogensis Engl. & $800-1400$ & $500-800$ & sandy soil & & & 12457 & & & $\begin{array}{l}\text { Zambia, } \\
\text { Zimbabwe }\end{array}$ \\
\hline $\begin{array}{l}\text { C. unilobata Gillett } \\
\text { and Vollesen }\end{array}$ & $70-1000$ & $200-350$ & alluvium soil & SD & 147 & & & $\mathrm{C} 2 \mathrm{~S} 1 \mathrm{~S} 2$ & \\
\hline C. velutina Chiov. & $400-900$ & $230-300$ & $\begin{array}{l}\text { sandy soil, } \\
\text { limestone } \\
\text { hills, old lava } \\
\text { flow }\end{array}$ & $A F, S D, H A$ & 1 & & & $\begin{array}{l}\mathrm{N} 1-3 \mathrm{C} 1-2 \\
\mathrm{~S} 1 \mathrm{S3}\end{array}$ & \\
\hline C. zanzibarica Engl. & $2-510$ & $900-1050$ & $\begin{array}{l}\text { coral rocks } \\
\text { near sea \& } \\
\text { streams }\end{array}$ & & 7 & & & & $\begin{array}{l}\text { Mozambique, } \\
\text { Malawi, } \\
\text { Zimbabwe, S. } \\
\text { Africa }\end{array}$ \\
\hline
\end{tabular}




\section{Teshome Soromessa}

Arabia (with six species of the Horn currently occurring in Arabia) to India (one species of the Horn which was cultivated occurs there) and are represented today by species such as $C$. wightii (synonym of the then $C$. mukul) and $C$. berryi. Further more species that currently occur in India include C. caudata, C. agallocha, C. pubescens and C. myrrha (Chithra and Henry, 1997).

The transoceanic distribution of Commiphora from the mainland in Madagascar, India and Arabia on one side and to Latin America on the other side is another remarkable feature of the group to be portrayed here. In connection with phytogeography the land bridge theory has been stipulated by several authors, notably van Steenis (1962), Wild (1965), Takhtajan (1969), Raven and Axelrod (1974), Schuster (1976), Turner (1995), RidderNuman (1996) and Haegens (2000) including literatures cited therein.

According to Raven and Axelrod (1974) and literatures cited therein, the separation of Africa from South America seems to have commenced 125-130 m.y. BP. The final marine connection associated with the spreading apart of Africa and South America took place slightly less than 100 m.y. BP with the continent remaining in near contact along strike-slip faults until at least 90 m.y. BP, when northeast Brazil and Africa were separated by only a narrow strait. The same authors adopted the concept that Africa and Europe were connected via Spain, in Early Paleocene (63 m.y BP). Africa may have been connected with Asia through Arabia at this time. Finally they came to the notion that from the Early Paleocene into the Upper Eocene (53 m.y. $\mathrm{BP})$, Africa and Europe seem to have become more widely separated. Furthermore Schuster (1976) and literatures cited therein suggested that until at least 75 million (roughly Late Cretaceous) years ago there were archipelagic connections between the Indian plate, the Mascarene Plateau, Madagascar and Africa. During this same period, India and Australia continued to move northward towards Eurasia, opening the Indian Ocean and beginning to close the Tethys Ocean. As India separated from the southeast coast of Africa it pulled Madagascar in its wake.

Following this plate tectonic events, the pattern of evolution and distribution of organism has been considered as events of great importance. Raven and Axelrod (1974) indicated that direct migration of organism between Africa, Madagascar (probably also India) and South America last possible about 100 m.y. BP and also reestablishment of direct connection between Africa and Eurasia was about 17 m.y. BP. Rabinowitz et al., (1983) and literatures cited therein, attested that the magnetic data point

\section{Sci. Technol. Arts Res. J., July-Sep 2013, 2(3): 93-104}

to the motion of Madagascar relative to Africa being from the north, with the Africa-Madagascar separation started beginning during the time of the Jurassic quite zone ( $165 \mathrm{Ma})$ and ending at a time of formation of anomaly M9 ( 121 Ma) denoting that the Africa-Madagascar separation began at about the same time of as the breakup of Gondwanaland and the separation of North America from Africa. On the other side, Storey et al., (1995) dated the separation of Madagascar and India before $~ 88$ Ma. However, recent studies of the Mozambique channel, revealed the possibility of the land bridge connecting mainland Africa and Madagascar from mid-Eocene to early Miocene (45-26 Ma) (McCall, 1997 as cited in Möller \& Cronk, 2001). On the other hand authors like Coleman et al., (1992), Tadiwos Cherinet et al., (1998) and Ukstins et al., (2002) dated the separation of Africa-Arabia to 30$20 \mathrm{Ma}$ range. From the above geological dating of separation events, one can come to the conclusion that the separation of Madagascar predates that of Arabia from Africa. This geological episode can be augmented by the presence of 6 Commiphora species in Arabia that also occur in the Horn as opposed to the endemic species of Commiphora in Madagascar. This shows that the Arabian species of Commiphora migrate recently to Arabia than to the rest of the world which still is in favor of the geological time of the separation of the two continents.

Correlation of continental separation with that of the fossil evidence might be the best evidence for the biogeography of a given group. However, the fossil records of a tropical family, Burseraceae, is very scanty with the available ones most from north temperate zone latitude, i.e. a little has changed since Muller (1981) suggested "at present only doubtful records of rare bureseraceous pollen grains have been published, interpretation of which will depend on a future detailed study of the recent pollen morphology of this family." Nevertheless, Graham \& Jarzen (1969) had recorded Bursera simaruba type pollen from Puerto Rico as Oligocene communities.

Furthermore, from the Eocene of Panama, Graham (1985) described Tetragastris-like pollen and Protium-like pollen, but this pollen (Protium-like) is considered as much more like Chrysophyllum L. pollen by Harley \& Daly (1995). Taylor (1990) also reported 6-7 putative genera of the family Burseraceae which are of Paleocene to upper Eocene age which include pollen of putative Protium and Tetragastris and the leaves of putative Bursera showing that the probable age of the family with much reservation, from this scanty information, lies between Paleocene-Eocene. 
Teshome Soromessa

Taking the separation of the continents and the meager information on the age of the family into consideration one could possibly assume that Commiphora migrate from the Horn of Africa to the different African regions, to Madagascar via the land bridge that was supposed to connect Africa and Madagascar, to Arabia and might or might not reach India. The recent report on the displacement of Bursera leptophloeos Mart. from Bursera to Commiphora leptophloeos (Mart.) Gillett comb. nov. of Commiphora Gillett (1980) indicated the existence of Commiphora species in Latin America. It is then impractical to assume that either Commiphora migrated from Africa to Latina America or from Latin America to Africa, for the fact that the separation of these two continents predates the evolution and diversification of most angiosperm families. One hypothetical explanation on the existence of Commiphora in Latina America and India might be linked to polyphyletic origin (note that the present study didn't consider the whole genus to confirm whether it is polyphyletic or monophyletic in origin) of Commiphora and that Commiphora might have derived from Bursera itself which is currently distributed in both places. The endemicity of the Madagascan species of Commiphora might suggest the possible speciation events in the area.

Wild (1965) on the other hand indicated that there are double patterns of distribution of Commiphora species: his first argument was that the occurrences of interpluvial periods in Africa produced xerophytic species that migrated to Arabia and India. His second argument for the notion was that the Lemurian land-bridge played its role since the more mesophytic species in one of the more
Sci. Technol. Arts Res. J., July-Sep 2013, 2(3): 93-104

favorable pluvial periods migrated along the Lemurian bridge into Madagascar with its twenty or so species and also into India and Ceylon. The morphological resemblances of $C$. wightii to that of the section containing Commiphora (which contains C. quadricincta, a northern species and $C$. madagascarensis, C. oblongifolia and C. ovalifolia the Tanzanian species) may suggest a two way migration of species that go with Wild's (1965) notion.

In contrast to the different kinds of colonization event, another possible explanation of the distribution of species across the continent is long distance dispersal. Even if this process might happen (animal dispersal in this case) in the past, the viability of the seeds of Commiphora species is quite in question.

\section{Vegetation, Altitudinal \& Pedological Preference}

Most, if not all, species of Commiphora in the NE of Africa occur in the southern and southeastern parts of Ethiopia. These include the lowlands of Hararge (HA in Figure 1), Bale (BA), Sidamo (SD), and some parts of Gamo Gofa (GG), with a high species concentration of Commiphora in Sidamo. Similarly, the East African species of Commiphora are highly concentrated in the Northern Frontier Province and the coast of Kenya in $\mathrm{K} 1$ and $\mathrm{K} 7$ as recognized in Flora of Tropical East Africa. High concentration of species occurs in T5 and T6 of Tanzania and U1 (around the Karamoja district of Uganda). Figure 4 shows comparison of the different flora regions in terms of the species abundances within the different political boundaries.

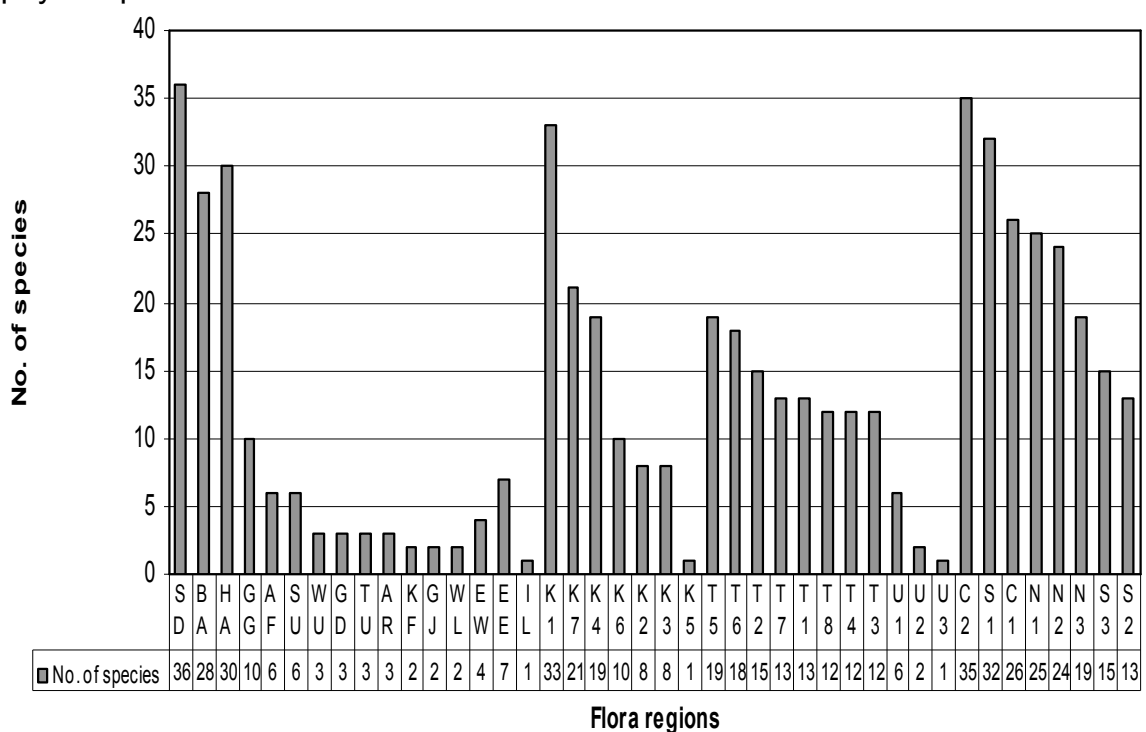

Figure 4: Comparison of different flora regions in terms of abundances of Commiphora species. The Flora regions are as recognized in the Flora of Ethiopia, Flora of Somalia and Flora of Tropical East Africa. Note also C2 of Somalia has high species concentration. 


\section{Teshome Soromessa}

The vegetation of these areas, although not studied deeply, have been classified and mapped by various authors. The physiognomic study made by Pichi-Sermolli (1957) resulted in the recognition of 24 vegetation units in his publication on the geobotany of Ethiopia, Eritrea, Djibouti and Somalia. Of 24 vegetation units recognized by Pichi-Sermolli (1957), unit 2, 3, 4, 6, 7, 11, 12 and 13 were considered to, at least, occur in one or more areas of the lowlands of Hararge, Sidamo, Bale and Gamo Gofa. However, some of his units, particularly unit 6, 7 and 11 are generalized ones and could not exactly tell the nature of vegetation in the areas.

Breitenbach (1963) studied the vegetation of Ethiopia based on physiognomy in relation to altitude and humidity and came up with seven vegetation types including various sub-types. In this study he mapped the lowlands of Hararge, Bale,
Sci. Technol. Arts Res. J., July-Sep 2013, 2(3): 93-104

Sidamo and Gamo Gofa under his lowlandwoodland, lowland-savannahs and lowland-steppes units. Although Breitenbach (1963) identified seven major units, the borders drawn on the vegetation maps produced from his study is quite similar to the one produced by Pichi-Sermolli's (1957). The next comprehensive study of African vegetation was that of White (1983). According to White (1983) southern and southeastern Ethiopia belongs to the SomaliMasai Regional Center of Endemism, one of his major units within the flora of Africa as a whole. It is the home of Commiphora and Acacias extending down to Somalia, Kenya, Tanzania and Uganda. In this regional center different plant communities could be encountered. This includes CommiphoraAcacia, Commiphora woodland (as in the case of pure Commiphora baluensis woodland in El Siro area, Figure 5) and or Commiphora bushland.

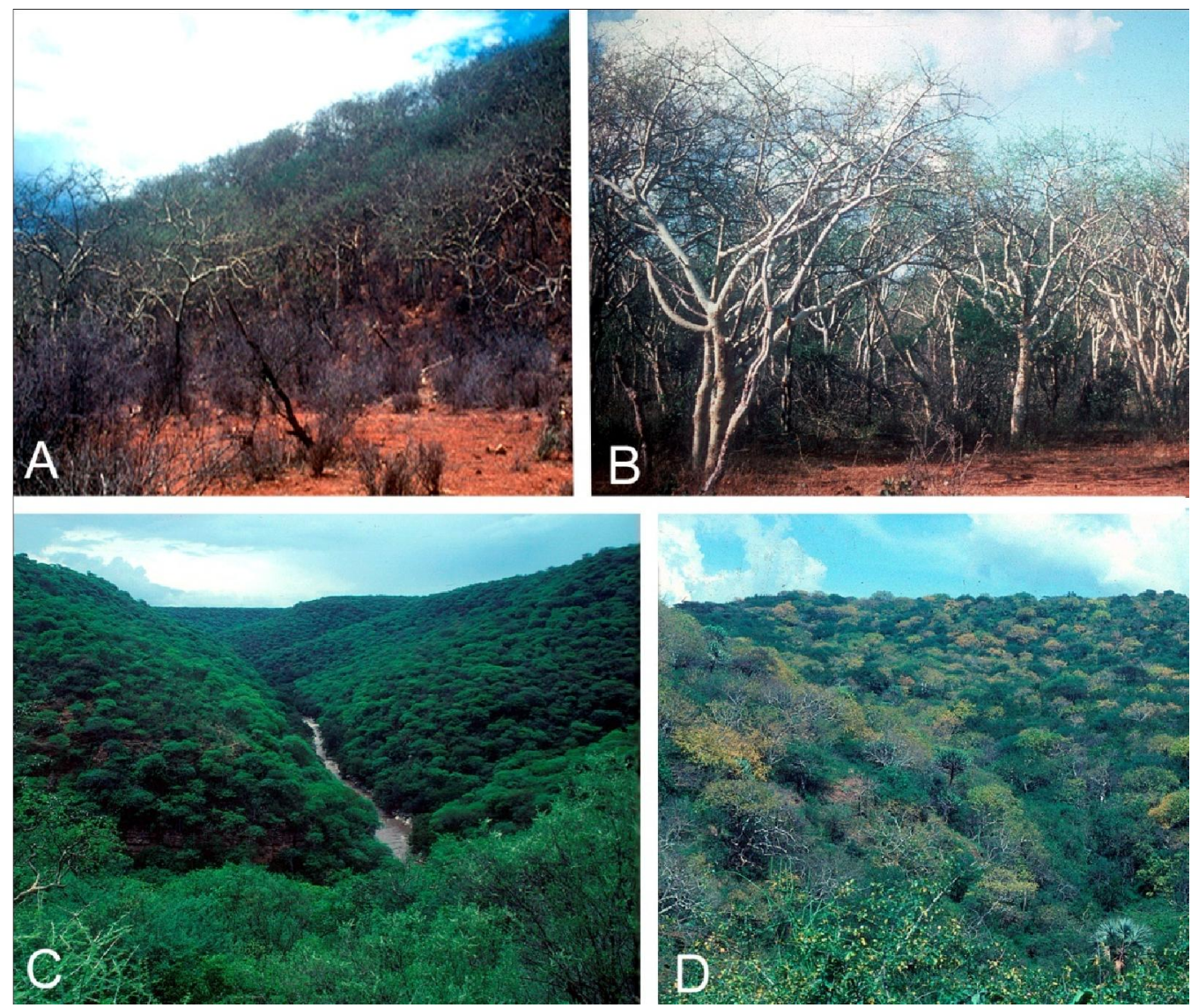

Figure 5: Some vegetation formations with Commiphora species. A-B, C. baluensis woodland; C, AcaciaCommiphora woodland around Sof Umar; Weyib river in the center; D, dry Commiphora woodland.

Generally speaking areas harboring Commiphora, belong to deciduous woodland and/or bushland usually conspicuously rich in species of Acacia and Commiphora. Associated with these vegetation types, there are extensive grassland areas that are found at relatively higher altitudes, notably at Nagelle and Jijiga, which probably were formed as a result of anthropogenic influences in the past years. Altitudinally Commiphora occur from almost sea level to about $2000 \mathrm{~m}$ above sea level 
Teshome Soromessa

(Table 1), on alternatively sandy soils, rocky hills, limestone, gypsum and sometimes on black cotton soils.

Further categorization of species based on the width of altitudinal niche they occupy shows that some species perform best in a wide range of altitude exploiting broader ecological niches while others preferably occur in a narrow range. Of all the
Sci. Technol. Arts Res. J., July-Sep 2013, 2(3): 93-104

species considered here for investigation, 20 species occur within a $500 \mathrm{~m}$ altitudinal range showing narrow range of performances (e.g., $C$. acuminata, C. chaetocarpa, C. gileadensis, C. erosa and $C$. suffruticosa, Table 2) and 7 species occupy altitudinal range exceeding $1500 \mathrm{~m}$ utilizing all opportunity from lowland, premontane to low montane range and successfully invading the habitat.

Table 2: Categorical performance of species in terms of altitudes.

\begin{tabular}{ccl}
\hline $\begin{array}{c}\text { Ranges of altitudinal } \\
\text { performance }\end{array}$ & $\begin{array}{c}\text { Number of } \\
\text { species }\end{array}$ & Examples of species in that category \\
\hline Within $500 \mathrm{~m}$ range & 20 & C. acuminata, C. erosa, C. suffruticosa, etc., \\
Within $500-1000 \mathrm{~m}$ range & 29 & C. alaticaulis, C. albiflora, . ciliata, etc., \\
Within $1000-1500 \mathrm{~m}$ range & 17 & C. corrugata, C. gurreh, C pedunculata, etc., \\
Above $1500 \mathrm{~m}$ range & 7 & C. africana, C. edulis, C. schimperi, etc., \\
\hline
\end{tabular}

Commiphora are aridisols species, a soil type known to occur in dry areas in general and in lowlands of Oromia, Ogaden and southern Ethiopia in particular (Mesfin Abebe, 1998). Some species of Commiphora occur preferably in the woodland vegetation on brown soils, black cotton soils and on a rocky lava places. They form association with Acacia, Boswellia, Combretum, Terminalia, Barbeya and some species of Lannea. Some of these species of Commiphora include Commiphora edulis, C. habessinica, C. pedunculata, C. engleri, $C$. serrulata and $C$. gurreh with $C$. pedunculata being an exception as it also occurs in wooded grassland vegetation. Other species of Commiphora tend to occur in bushland vegetation forming an extensive association with Acacia and some Acanthaceae. Some notable species to this group are $C$. acuminata, C. albiflora, C. campestris, C. ellenbeckii, C. horrida, C. sphaerocarpa, $C$. staphyleifolia and $C$. unilobata. The bushland Commiphora largely occur on rocky lime stone hills. Other than the bushland or woodland species, some are widespread over both bushlands and woodlands frequently occupying sandy soil that overlay limestone. These species include the widespread $C$. africana, $C$. baluensis and $C$. schimperi. Other widespread species include Commiphora boranensis, C. confusa, C. corrugata, $C$. cyclophylla, C. monoica, C. myrrha and C. rostrata. Commiphora corrugata, C. baluensis and C. rostrata also occur in wooded grassland vegetation, which is primarily dominated by grass. Interestingly, Commiphora corrugata and C. hildebrandtii are species that often perform best with Combretum molle and Terminalia brownii.

\section{Implications for in-situ Conservation}

Commiphora form important and major parts of the vegetation in what is known as White's (1983)
Somalia-Masai Regional Center of Endemism. It is also the best sources of browse for the large livestock of the region. The need for trees that can be browsed coupled with the need for arable land in the areas may lead to degradation of their genetic diversity. Alluding to earlier discussion on the diversity of Commiphora species, flora regions like $\mathrm{SD}, \mathrm{HA}, \mathrm{BA}, \mathrm{K} 1, \mathrm{~S} 1, \mathrm{C} 2$ and $\mathrm{C} 1$ are regions of high genetic pool. Identification of rich centers of diversity is then important for undertaking in-situ conservation of the genetic resources. Regions with high diversity of Commiphora species could serve as a possible in-situ conservation site. Although considering all these regions for in-situ conservation seems to be expensive, regions that are endowed with greater genetic pool can be considered for conservation purposes. Good examples are Sof Umar in Bale and Walensu Ranch in Sidamo where 12 and 13 species of Commiphora have been recorded. Similar gene conservation activities can be made in the other regions having high genetic diversity of Commiphora species.

\section{CONCLUSIONS}

Commiphora are pan tropical in distribution performing well in arid and semi-arid environments occupying an ecological range between $1-2100 \mathrm{~m}$ above sea level striving best on aridisols. More over, some species of the genus tend to perform well in a narrow ecological range. Results from the phytogeographic analysis of the group revealed three patterns of distributions: species with wider distribution ranges, endemic and transgressing species having limited niche and those found in flora regions that are appropriate environment for Commiphora to diversify and invade the regions. 
Teshome Soromessa

Although tracing the migratory route and possible origin of the group requires numerous information, studies such as Wild (1965) and Teshome (2005) suggested that the possible origin of Commiphora seems to be in the Horn of Africa. Based on the separation of the continents and the meager information on the age of the family into consideration it is possible to assume that Commiphora migrate from the Horn of Africa to the different African regions, Madagascar and Arabia. It seems practical to note that Commiphora did not migrate to Latin America nor vice versa, since the separation of African and Latina American continents predates the evolution and diversification of most angiosperm families showing that Commiphora might have polyphyletic origin. However, the migratory route of Commiphora still requires more data, particularly complete data of Commiphora of the world. Data on the age of the genus is also important to corroborate it with the plate tectonic theory.

\section{REFERENCES}

Berg, O. (1862). Die Balsamodendron-Arten der Berliner Herbarien. Botanische Zeitung 21: 163-165.

Boulos, L. (2000). Burseraceae. Flora of Egypt, Geraniaceae-Boraginaceae (ed. by Boulos) 2: 68-69. Al Hadara Publishing, Cairo, Egypt.

Breitenbach, F. Von. (1963). The Indigenous Trees of Ethiopia, $2^{\text {nd }}$ edn. Ethiopian Forestry Association, Addis Ababa.

Brooks, D.R. (1990). Parsimony analysis in biogeography and coevolution: methodological and theoretical update. Systematic Zoology 39: 14-30.

Burtt, B.D. (1935). Observation on the genus Commiphora and its distribution in Tanganyika territory. Kew Bulletin 3: 101-117.

Chiovenda, E. (1932). Burseraceae. Flora Somala 2. Modena: R. Orto Botanico.

Chithra, V. \& Henry, A.N. (1997). Burseraceae. Flora of India, Malpighiaceae-Dichapetalaceae (eds. by Hajra, P.K., Nair, V.J. \& Daniel, P), 4: 431-452. Botanical Survey of India, Calcutta.

Coleman, R.G., DeBari, S. \& Peterman, Z. (1992). A-type granite and the Red Sea opening. Tectonophysics 204(1-2): 27-40.

Engler, A. (1898). Burseraceae Africanae II. Botanische Jahrbücher für Systematik, Pflanzengeschichte und Pflanzengeographie 26: 364-373.

Engler, A. (1904). Burseraceae Africanae III. Botanische Jahrbücher für Systematik, Pflanzengeschichte und Pflanzengeographie 34: 302-316.

Engler, A. (1910). Burseraceae Africanae IV. Botanische Jahrbücher für Systematik, Pflanzengeschichte und Pflanzengeographie 44: 137-155.
Sci. Technol. Arts Res. J., July-Sep 2013, 2(3): 93-104

Engler, A. (1912). Burseraceae Africanae V. Botanische Jahrbücher für Systematik. Pflanzengeschichte und Pflanzengeographie 46: 289-292.

Engler, A. (1913). Die Verbreitung der Afrikanischen Burseraceen im Verhältnis zu ihrer systematischen Gliederung und die Einteilung der Gattung Commiphora. Botanische Jahrbücher für Systematik, Pflanzengeschichte und Pflanzengeographie 48: 443490.

Engler, A. (1931). Burseraceae, Die Natürlichen Pflanzenfamilien (eds. by Engler, A. and Prantl, K.), 19a: 405-456.

Gillett, J.B. (1980). Commiphora (Burseraceae) in South America and its relations to Bursera. Kew Bulletin 34 (4): $569-587$.

Gillett, J.B. (1991). Burseraceae, Flora of Tropical East Africa (ed., by Polhill, R.M.), 1-94. A.A. Balkema, Rotterdam, Netherlands.

Graham, A. (1985). Studies in neotropical paleobotany IV. The Eocene communities of Panama. Annals of the Missouri Botanic Garden 72: 504-534.

Graham, A. \& Jarzen, D.M. (1969). Studies in neotropical paleobotany I. The Oligocene communities of Puerto Rico. Annals of the Missouri Botanic Garden 56: 308357.

Haegens, R. (2002). Taxonomy, phylogeny, and Biogeography of Baccaurea, Distichirhops, and Nothobaccaurea (Euphorbiaceae). Blumea 12: 1-218.

Harley, M.M. \& Daly, D.C. (1995). Burseraceae Kunth, Protieae March. em. Engl. World Pollen and Spore Flora 20: 1-44.

Hedberg, I. \& Edwards, S. (eds.) (1989). Flora of Ethiopia, Vol. 3. Pittosporaceae to Araliaceae. The National Herbarium, Addis Ababa University, Ethiopia \& Department of Systematic Botany, Uppsala University, Sweden.

Leenhouts, P.W. (1956). Burseraceae, Flora Malesiana (ed. by Van Steenis, C.G.G.J.), 5 (2): 209-296. Noordhoff-Kolff N.V., Djakarta.

Mesfin Abebe. (1998). Nature and Management of Ethiopian Soils. Published by Alemaya University of Agriculture, Ethiopia.

Möller, M. \& Cronk, Q. C. B. (2001). Phylogenetic studies in Streptocarpus (Gesneriaceae): reconstruction of biogeographic history and distribution pattern. Systematic Geography of Plants 71: 545-555.

Muller, J. (1981). Fossil pollen records of extant angiosperms. Botanical Review 47: 1-142.

Pichi-Sermolli, R.E.G. (1957). Una carta geobotanica dell' Africa Orientale (Eritrea, Etiopia, Somalia). Webbia 13: 15-132.

Polhill, D. (1988). Flora of Tropical East Africa, Index of collection localities. Royal Botanic Gardens, Kew, Whitstable Litho Printers, Ltd. Great Britain. 


\section{Teshome Soromessa}

Rabinowitz, P.D., Coffin, M.F. \& Falvey, D. (1983). The Separation of Madagascar and Africa. Science 220: 67-69.

Raven, H.P. \& Axelrod, I. D. (1974). Angiosperm Biogeography and past continental movements. Annals of the Missouri Botanic Garden 61: 539-673.

Ridder-Numan, J. W. A. (1996). Historical Biogeography of Southeast Asian genus Spatholobus (LegumPapilionoideae) and its allies. Blumea 10: 1-144.

Schuster, R.M. (1976). Plate tectonics and its bearing on the geographical origin and dispersal of Angiosperms. Origin and Early Evolution of Angiosperm (ed. by Beck, C. B.), 48-138. Columbia University Press, New York.

Sprague, T. A. (1927). Commiphora spp. Hooker's Icones Plantarum, 2 t: 3105-3112.

Storey, M., Mahoney, J.J., Saunders, A.D., Duncan, R.A., Kelley, S.P. \& Coffin, M.F. (1995). Timing of Hot SpotRelated Volcanism and Breakup of Madagascar and India. Science 267: 852-855.

Swofford; D. L. (2002). PAUP*. Phylogenetic Analysis Using Parsimony (* and other methods). Version 4. Sinauer Associates, Sunderland, Massachusetts.

Tadiwos Cherinet, Hart, W.K., Aronson, J.L. \& Walter, R.C. (1998). New age constraints on the timing of volcanism and tectonism in the northern Main Ethiopia Rift-southern Afar transition zone (Ethiopia). Journal of Volcanology and Geothermal Research 80: 267-280.

Takhtajan, A. (1969). Flowering plants origin and dispersal. Oliver \& Boyd: Edinburgh.

Tyalor, D.W. (1990). Paloebiogeogarphic relationships of Angiosperms from Cretaceous and Early Tertiary of the North American area. Botanical Review 56: 279417.

Teshome Soromessa (2005). Phylogentic study of NE and E Tropical African Commiphora species. Ethiopian Journal of Biological Science 4 (1): 27-58.

Teshome Soromessa (2006). A new species of Commiphora (Burseraceae) from Ethiopia. Ethiopian Journal of Biological Sciences 5(1): 1-8.
Sci. Technol. Arts Res. J., July-Sep 2013, 2(3): 93-104

Thulin, M. (1999). Burseraceae, Flora of Somalia, Tiliaceae-Apiaceae (ed., by Thulin, M), 2: 183-228. Royal Botanic Gardens, Kew.

Turner, H. (1995). Cladistic and biogeographic analysis of Arytera Blume and Mischarytera gen. nov (Sapindaceae) with notes on methodology and a full taxonomic revision. Blumea 9: 1-230.

Ukstins, I.A., Renne, P.R., Wolfenden, E., Baker, J., Dereje Ayalew \& Menzies, M. (2002). Matching conjugate volcanic rifted margins: ${ }^{40} \mathrm{Ar} /{ }^{30} \mathrm{Ar}$ chronostratigraphy of pre- and syn-rift bimodal flood volcanism in Ethiopia and Yemen. Earth Planet and Science Letter 198: 289-306.

van der Walt, J.J.A. (1973). The South African species of Commiphora. Bothalia 11 (1 \& 2), 53-102.

van der Walt, J.J.A. (1975). The fruit of Commiphora. Boissiera 24: 325-330.

van der Walt, J.J.A. (1986). Burseraceae, Flora of Southern Africa, Simaroubaceae-Malpighiacaea (ed. by Leistner, O.A.), 18 (3): 5-34. Botanical Research Institute, Department of Agriculture and Water Supply, Republic of South Africa.

Van Steenis, C.G.G.J. (1962). The land bridge theory in Botany with practical references to tropical plants. Blumea 11 (2): 235-372.

White, F. (1983). The vegetation of Africa. A descriptive memoir to accompany the UNESCO/AETFAT/UNSO vegetation map of Africa. UNESCO, Paris.

Wild, H. (1959). A revised Classification of the Genus Commiphora Jacq. Boletim Sociedade Broteriana 33 67-95.

Wild, H. (1963). Burseraceae, FI. Zambia (eds. by Exell, A.W., Fernandes, A. \& Wild, H.), 2 (1): 263-285. Crown Agents for oversea Governments and Administrations, London.

Wild, H. (1965). Additional evidence for the AfricanMadagascar-India-Ceylon Land-Bridge Theory with special reference to the genera "Anisopappus" and Commiphora. Webbia 19: 497-505. 